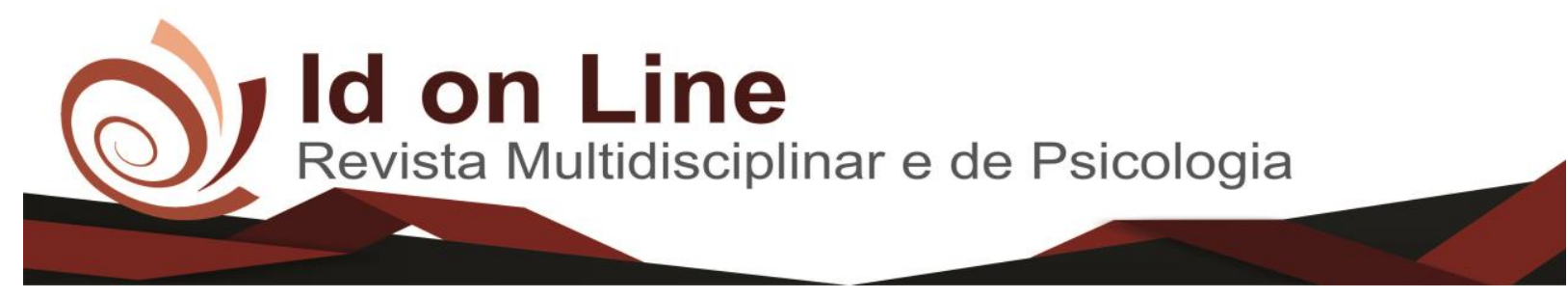

Artigo

\title{
Ação e eficácia do tratamento com a Radiofrequência na adiposidade abdominal em mulheres
}

Susan Caroline Santos Ferreiral; Karla Cavalcante Silva de Moraes²; Jessica Almeida Barros; Jussara Santos de Souza ${ }^{4}$; Beatriz Souza Carvalho Santos ${ }^{5}$; Juliana Barros Ferreira ${ }^{6}$

\begin{abstract}
Resumo: O objetivo deste estudo foi avaliar a ação e influência do tratamento da radiofrequência na adiposidade abdominal e analisar a imagem corporal após o tratamento com a radiofrequência. Trata-se de um estudo experimental, realizado em 11 mulheres com aplicação de um questionário sociodemográfico e do body shape questionnaire para análise da imagem corporal. O tratamento foi realizado com a radiofrequência, utilizando uma temperatura entre $36^{\circ}$ a $38^{\circ} \mathrm{C}$, por 10 sessões, 2 vezes por semana. Os dados foram analisados de forma descritiva e o teste de t-student para comparação das variáveis. Constatou-se que houve uma redução da medida perimétrica supraumbilical $(\mathrm{p} \leq 0,005)$ e da medida perimétrica infraumbilical $(\mathrm{p} \leq 0,0004)$. Na adipometria, a diferença média após o tratamento foi de $2,96(\mathrm{p}<0,05)$. A radiofrequência no tratamento da adiposidade abdominal mostrou ser eficaz e proporcionou uma melhor imagem corporal.
\end{abstract}

Palavras-chave: Adiposidade Abdominal. Imagem corporal. Mulheres. Radiofrequência.

\section{Action and efficacy of radiofrequency treatment in abdominal adiposity in women}

\begin{abstract}
The aim of this paper was to evaluate the action and influence of the radiofrequency treatment in abdominal and analyze the body image after treatment with radiofrequency. This is an experimental study carried out on 11 women with a sociodemographic questionnaire and the body shape questionnaire for body image analysis. The treatment was performed with radiofrequency, using a temperature between $36^{\circ}$ to $38^{\circ} \mathrm{C}$, for 10 sessions, twice a week. The data were analyzed descriptively and the t-student test was used for comparison of variables. It was found that there was a reduction of the supraumbilical perimetric measurement $(\mathrm{p} \leq 0.005)$ and the infraimbilical perimetric measurement $(\mathrm{p} \leq 0.0004)$. In adipometry, the average difference after treatment was 2.96 ( $\mathrm{p}<0.05$ ). Radiofrequency in the treatment of abdominal adiposity was effective and provided a better body image.
\end{abstract}

Keywords: Abdominal Adiposity. Body image. Women. Radiofrequency.

\footnotetext{
1,3,4,5 Discente graduanda do curso de Fisioterapia pela Faculdade Independente do Nordeste-FAINOR. http://www.fainor.com.br, Vitória da Conquista - BA, Brasil.

${ }^{2}$ Fisioterapeuta. Docente da FAINOR e Maurício de Nassau. Mestre em Saúde Pública-ENSP/FIOCRUZ. Vitória da Conquista-BA, Brasil.

${ }^{6}$ Fisioterapeuta, Docente da FAINOR e FTC em Vitória da Conquista/BA. Mestre em Tecnologias em Saúde pela Escola Bahiana de Medicina e Saúde Pública, Salvador/Ba. Pesquisadora do Centro de Atenção ao Assoalho PélvicoCAAP em Salvador - BA, Brasil.
}

(*) Autor correspondente: Juliana Barros Ferreira, Rua Sifredo Pedral Sampaio, 414; Bairro Recreio; CEP: 45020-190; Vitória da Conquista/BA; julibarros78@ hotmail.com (77)98819 - 2774. 


\section{Introdução}

A imagem corporal é a forma como cada indivíduo pensa de si mesmo e como percebese fisicamente, ou seja, uma autoimagem. Esta percepção pode ser modificada de acordo com a idade e é influenciada por padrões socioculturais a manter um estereotipo de corpo perfeito (ARENAS; MARTÍNEZ, 2015). Um dos fatores que promove essa grande incidência da busca pelo corpo perfeito é a mídia, e os seus padrões de beleza impostos. Isto favorece e proporciona a não aceitação da forma física, e gera interferência na imagem corporal (MARTINS; PETROSKI, 2015).

No Brasil já foram realizados mais de 2,5 milhões de procedimentos estéticos, sendo estes cirúrgicos ou não, ficando atrás apenas para os Estados Unidos que apontou cerca de três milhões (FERREIRA; LEMOS; SILVA, 2016). Por conta desta busca por um corpo perfeito indivíduos tentam de todas as formas lapidarem a sua aparência corporal, ao passo que o aumento da gordura corporal, particularmente a localizada na região abdominal, colabora para a baixa autoestima (SILVA et al., 2014).

Existe uma grande demanda de indivíduos buscando formas de tratamentos que promovam uma melhora estética de maneira rápida e com risco mínimo, e por conta disso surgem os procedimentos não cirúrgicos. Dentre estes, existe uma variedade de aparelhos e recursos de alta tecnologia como opções terapêuticas seguras e eficazes, e um destes tratamentos, que atua de maneira não invasiva, é a radiofrequência (RF) (TAGLIOLATO, 2015).

A RF gera calor no tecido subcutâneo e tem por finalidade tratar a adiposidade, que é definida por um processo irregular do tecido conjuntivo subcutâneo, onde os adipócitos apresentam-se aumentados em determinadas regiões específicas causando uma deformidade tecidual. Este efeito térmico irá ocasionar um processo de degradação dos adipócitos, levando a redução de medidas e proporcionando uma melhora no processo de reorganização das fibras de colágeno. (LOFEU et al., 2015).

Portanto, através do exposto, a adiposidade abdominal pode ser caracterizada como uma afecção incômoda e indesejável, por isso faz-se necessário analisar os efeitos da RF no tratamento da mesma, verificando se há uma melhora na imagem corporal dos indivíduos. Tal informação irá contribuir para o conhecimento de acadêmicos, profissionais e pessoas que 
desejam realizar o procedimento. Desta forma, o presente estudo objetivou avaliar a ação e influência antes e após o tratamento da RF na adiposidade abdominal e imagem corporal dos indivíduos.

\section{Metodologia}

Trata-se de um estudo experimental, analítico, de corte transversal e de caráter quantitativo. A pesquisa foi realizada no Núcleo de Estudo em Fisioterapia (NEF) de uma faculdade privada, situada no município de Vitória da Conquista - BA.

Os dados foram coletados no período de julho a setembro de 2017, nos turnos matutino e vespertino, duas vezes por semana, utilizando método de abordagem por conveniência. A população foi constituída por 11 mulheres estudantes de um curso da área de saúde, que aceitaram participar do estudo, para tratamento da adiposidade em região abdominal através da aplicação da Radiofrequência.

Os critérios de inclusão foram estudantes do sexo feminino, com idade entre 18 a 25 anos, que apresentassem lipodistrofia em região abdominal. E os critérios de exclusão foram acadêmicas não praticantes de atividade física, com alguma patologia hematológica, doença crônica degenerativa ou neurológica, em uso de marca-passo, de dispositivo intrauterino (DIU), portadores de neoplasias e gestantes.

As participantes receberam um questionário sociodemográfico, que identificou raça, estado civil e renda familiar. Ao início e final do tratamento foi aplicado o questionário Body Shape e realizadas as medidas de perimetria e adipometria abdominal. É válido destacar que estes procedimentos foram realizados em uma sala privada na presença do pesquisador e da profissional especializada.

O questionário Body Shape, sobre a Imagem Corporal (Body Shape Questionnaire BSQ) é um teste de autopreenchimento com 34 perguntas para serem respondidas segundo a escala LIKERT de 1 a 6 , onde (1- quer dizer nunca, 2 - raramente, 3 - às vezes, 4 frequentemente, 5 - muito frequentemente, 6- sempre). De acordo com a resposta marcada, o valor do número correspondente à opção feita é computado como ponto para a questão (por exemplo: nunca, vale um ponto). O total de pontos obtidos no instrumento é a soma de cada 
resposta marcada e reflete os níveis de preocupação com a imagem corporal. Obtendo resultado menor ou igual a 110 pontos, é constatado um padrão de normalidade e tido como ausência de distorção da imagem corporal. Resultados entre 110 e 138 pontos é classificado como leve distorção da imagem corporal; entre 138 e 167 é classificado como moderada distorção da imagem corporal; e acima de 167 pontos a classificação é de presença de grave distorção da imagem corporal (DI PIETRO, 2001).

Logo após, aplicou-se a ficha de avaliação fisioterapêutica dermatofuncional, com a identificação da participante, telefone para contato, número de sessões, data das sessões, temperatura cutânea, potência inicial e final, tempo de duração da sessão, perimetria supra e infraumbilical e adipometria cutânea da borda lateral da cicatriz umbilical à direita.

A perimetria foi realizada com fita métrica marca ISP (Instituto São Paulo), onde a mesma foi posicionada a $2,5 \mathrm{~cm}$ respectivamente, acima e abaixo da cicatriz supra e infraumbilical. Após a realização desta perimetria realizou-se a adipometria com o adipômetro científico da marca Sanny AD 1010 posicionado na prega vertical à direita da cicatriz umbilical com distância de 2,5 cm da região supracitada (BORGES; SCORZA, 2016).

Foram realizadas 10 sessões de RF, duas vezes na semana. A aplicação da RF foi realizada por uma graduanda em fisioterapia, por meio de aparelho da marca HTM®, configuração bipolar e o método de transferência não ablativa, com intensidade inicial de 50\% e frequência de 1,2 MHz. Para aplicação utilizou-se gel glicerinado manipulado. As participantes foram instruídas a não usar nenhum tipo de loção hidratante ou óleo na região antes da aplicação da RF. As mesmas foram posicionadas em decúbito dorsal na maca de marca ISP (Instituto São Paulo) e antes de cada aplicação era realizada a assepsia da região com álcool 70\% da marca Santa Cruz. A região abdominal foi demarcada em áreas (AGNE, 2016). Utilizou-se a manopla ou ponteira corporal concêntrica de transferência capacitiva com movimentos circulares na região abdominal. Simultaneamente à movimentação do eletrodo, mensurou-se a temperatura por um termômetro infravermelho. Ao se atingir a temperatura entre $36^{\circ}$ a $38^{\circ} \mathrm{C}$, o movimento foi mantido nessa temperatura por mais dois minutos. Cada sessão obteve duração média de 20 minutos.

O parâmetro absoluto respeitado foi a tolerância da paciente, diminuía-se a intensidade aplicada, quando referido qualquer desconforto na região da aplicação. 
As participantes foram devidamente esclarecidas quanto aos riscos benefícios e objetivos da pesquisa, ficando livres para participarem ou não. Ao aceitarem, assinaram o Termo de Consentimento Livre e Esclarecido (TCLE), respeitando os princípios éticos que constam na resolução 466/12 do Conselho Nacional de Saúde para que os dados possam ser utilizados e os resultados divulgados. Destaca-se que o projeto foi aprovado pelo Comitê de Ética em Pesquisa da Faculdade Independente do Nordeste sob o número do CAE: 68339517.9.0000.5578 e Parecer n 2.108.559.

A correlação entre as variáveis foram avaliadas utilizando teste de t-Student para amostras pareadas com uma significância de 0,05 (ou confiabilidade de 0,95). Para elaboração do banco de dados utilizou-se o aplicativo Microsoft Office Excel 2013 plus e para análise dos dados, o programa estatístico SPSS®, versão 22.0.

\section{Resultados}

A amostra foi composta por 11 mulheres, estudantes universitárias. Em relação ao estado civil 90,9\% eram solteiras e 54,5\% de raça parda. Ressalta-se ainda que, 81,8\% não possuem renda ou não sabem (Tabela 1).

Tabela 1 - Dados Sociodemográficos. Vitória da Conquista- BA, 2017.

\begin{tabular}{lllrr}
\hline Variáveis & \multicolumn{1}{c}{$\begin{array}{c}\text { \% } \\
\text { Estado civil }\end{array}$} & 100 & n & \% \\
& Solteira & & 10 & 90,9 \\
Raça & Casada & 100 & 1 & 9,1 \\
& & & & \\
& Branca & & 4 & 36,4 \\
Renda familiar & Negra & & 1 & 9,1 \\
& Parda & 100 & 6 & 54,5 \\
& Sem renda/não sabe & & 9 & 81,8 \\
& Até um salário mínimo & & 2 & 18,2 \\
\hline
\end{tabular}

Fonte: Dados da pesquisa 
A tabela 2 traz dados referentes à perimetria e a adipometria antes e após a aplicação da RF. Em relação aos resultados obtidos nas medidas de perimetria supraumbilical, a média anterior estava 81,00 \pm 7,33. Após a aplicação da RF diminuiu para 78,36 $\pm 6,69$, obtendo-se a diferença média de 2,64. Ao examinar á perimetria infraumbilical, observa-se a média de 91,45 $\pm 7,43$ antes e após aplicação $87,36 \pm 6,24$ com diferença média de 4,09. Havendo significância $(\mathrm{p}<0,05)$.

No que diz respeito à avaliação da adipometria percebe-se uma diferença média de 2,96, pois, antes da aplicação apresentava-se 22,96 $\pm 8,63$ e após $20,00 \pm 7,37$, apresentando significância $(\mathrm{p}<0,05)$.

Tabela 2. Perimetria e adipometria antes e depois da RF. Vitória da Conquista-BA

\begin{tabular}{lcccccc}
\hline Variáveis & \multicolumn{7}{c}{ Antes RF } & Depois RF & & \\
\hline & Média & DP $^{1}$ & Média & DP & Df-m & Sig. \\
\cline { 2 - 7 } Perimetria & & & & & & \\
$\quad$ Supraumbilical & 81,00 & $\pm 7,33$ & 78,36 & $\pm 6,69$ & 2,64 & 0,005 \\
$\quad$ Infraumbilical & 91,45 & $\pm 7,43$ & 87,36 & $\pm 6,24$ & 4,09 & 0,0004 \\
Adipometria & & & & & & \\
$\quad$ Borda lateral $^{4}$ & 22,96 & $\pm 8,63$ & 20,00 & $\pm 7,37$ & 2,96 & 0,0027 \\
\hline
\end{tabular}

${ }^{1}$ Desvio Padrão; ${ }^{2}$ Diferença média ; ${ }^{3}$ Significância pelo teste t-Student; ${ }^{4}$ Borda lateral da cicatriz umbilical à direita.

Fonte: Dados da pesquisa

A Tabela 3 traz a percepção da imagem corporal antes e depois da radiofrequência. Observa-se que antes da RF 45,5\% da amostra tinham leve distorção corporal e após a aplicação da RF houve predominância 72,7\% em ausência de distorção corporal.

Tabela 3. Imagem corporal antes e depois da RF. Vitória da Conquista- BA, 2017.

\begin{tabular}{|c|c|c|c|c|}
\hline \multirow{2}{*}{ Variáveis } & \multicolumn{2}{|c|}{ ANTES } & \multicolumn{2}{|c|}{ DEPOIS } \\
\hline & $\mathbf{N}$ & $\%$ & $\mathbf{n}$ & $\%$ \\
\hline Ausência de distorção corporal & 4 & 36,4 & 8 & 72,7 \\
\hline Leve distorção corporal & 5 & 45,5 & 2 & 18,2 \\
\hline Moderada distorção corporal & 2 & 18,2 & 1 & 9,1 \\
\hline
\end{tabular}

Fonte: Dados da pesquisa 


\section{Discussão}

O aumento da gordura na região abdominal afeta grande parte da população, onde os mesmos estão cada vez mais preocupados com sua aparência física. $\mathrm{Na}$ busca incessante de estarem dentro dos padrões de beleza impostos pela sociedade, indivíduos de modo geral, mas principalmente as mulheres, se submetem a uma série de tratamentos.

O presente estudo demonstrou em relação à perimetria e adipometria após as 10 sessões uma diminuição de forma significativa da adiposidade abdominal. No estudo de Pumprla et al. (2015) realizado com 20 indivíduos, na $4^{\circ}$ aplicação já pode-se observar redução da circunferência abdominal. Suh et al. (2016) realizou sessões de 45 minutos, por 12 semanas obtendo também redução. Estes resultados vão de encontro ao estudo de Boisnic et al. (2014) no qual houve redução da circunferência abdominal $(113,4-110,7 \mathrm{~cm})$ e da espessura do tecido adiposo subcutâneo (40,5-38,5 mm). Já no estudo de intervenção de Machado et al. (2017), onde o mesmo utiliza uma terapia não invasiva para adiposidade abdominal, houve uma maior redução de medidas principalmente na região infra umbilical, corroborando com o presente estudo.

Segundo Costa et al. (2009), a RF gera ondas de calor profundo, estas ondas possuem o potencial para gerar intenso calor e energia na camada mais profunda da pele, promovendo aquecimento dentro do tecido, gerando hiperemia, levando dessa forma um aumento da microcirculação sanguínea, atividade metabólica, térmica e enzimática gerando uma lipólise dos adipócitos, eliminando assim a gordura. Nesta mesma linha, Inácio, Bernardi e Romano (2017) destacam que a Radiofrequência possui indicação para todos os processos degenerativos que impliquem na diminuição ou retardo do metabolismo, irrigação e nutrição.

Segundo Tagliolatto (2015) a redução da gordura acontece, pois além de ocasionar o remodelamento do colágeno, o calor vai levar a uma melhor circulação sanguínea, produzindo efeitos eletrotérmicos na pele e na região subcutânea.

Em relação à imagem corporal, o estudo atual demonstrou que antes da aplicação da técnica com a RF houve leve distorção, e após a aplicação da técnica da RF em região abdominal, a predominância foi de ausência da distorção da imagem corporal.

A literatura não traz resultados associados da imagem corporal com a intervenção da RF. Porém, um estudo observacional, traz resultados significativos da imagem corporal. O 
estudo foi realizado no interior da Bahia e teve como objetivo comparar a imagem corporal, a qualidade de vida e satisfação com os tratamentos estéticos. Os resultados mostram que após os tratamentos realizados, a imagem corporal passou de leve distorção para um padrão de normalidade (FERREIRA; LEMOS; SILVA, 2016). Já um estudo realizado por Santana (2013), os adolescentes classificados pelo IMC como obesos ou com sobrepeso, apresentaram-se mais insatisfeitos com a percepção do peso e do corpo, além de estarem mais vulneráveis a atitudes negativas com a auto imagem.

Cria-se a hipótese que este resultado positivo e satisfatório da imagem corporal, pode estar relacionado ao fato do estudo ter sido realizado com mulheres jovens, pois as mesmas sentem-se incomodadas com a forma que as outras pessoas pensam ao olharem para elas. E isto proporciona uma busca constante à adaptação aos padrões de beleza impostos pela sociedade (ANDRADE et al., 2017).

As limitações deste estudo foram não ter tido um grupo controle e registros fotográficos para comparação dos resultados, além de não ter sido realizado o cálculo do índice de massa corpórea (IMC) das participantes.

\section{Considerações Finais}

Após a realização da pesquisa, conclui-se que a RF é uma técnica eficaz e tem ação no tratamento da adiposidade abdominal, comprovadas pela redução da perimetria e da adipometria abdominal, promovendo um resultado positivo na imagem corporal das mulheres.

Portanto, é possível afirmar que existe uma relação direta entre imagem corporal e os resultados da RF pois quando o indivíduo se sente satisfeito com sua imagem corporal, sua autoestima e seu bem-estar são elevados. Destaca-se ainda que estes resultados servem de embasamento cientifico para profissionais aplicarem a técnica com segurança e eficácia.

\section{Referências}

AGNE, J.E. Criolipólise e outras tecnologias no manejo do tecido adiposo. Santa Maria: Andreoli, 2016. 206 p. 
ANDRADE, I.S. et al. Associação entre a Percepção da Imagem Corporal com Indicadores Antropométricos em Adolescentes. Id on Line REVISTA DE PSICOLOGIA, v. 11, n. 35, p. 531-541, 2017.

ARENAS, J.J.S; MARTÍNEZ, A.O. Relationship between self-esteem and body image in children with obesity. Revista Mexicana de Trastornos Alimentarios, v. 6, n. 1, p. 38-44, 2015

BOISNIC, S. et al. A clinical and biological evaluation of a novel, noninvasive radiofrequency device for the long-term reduction of adipose tissue. Lasers in surgery and medicine, v. 46, n. 2, p. 94-103, 2014.

BORGES, F.D.S; SCORZA, F.A. Terapêutica em estética: Conceitos e Técnicas. São Paulo: Editora Phorte, 2016. 580 p.

COSTA, E.M. et al. Avaliacao dos efeitos do uso da tecaterapia na adiposidade abdominal. Kairós, 2009

DA SILVA, J.F. et al. A relação entre alterações posturais e gordura localizada: revisão de literatura. Revista Diálogos Acadêmicos, v. 3, n. 2, 2014.

DI PIETRO, M. C. Validade interna, dimensionalidade e desempenho da escala BSQ - "Body Shape Questionnaire" em uma população de estudantes universitários. 2001. 39 f. Dissertação (Mestrado em Ciências) - Escola Paulista de Medicina, Universidade Federal de São Paulo, São Paulo, 2001.

FERREIRA, J.B; LEMOS, L.M.A; DA SILVA, T.R. Qualidade de vida, Imagem corporal e satisfação nos tratamentos estéticos. Revista Pesquisa em Fisioterapia, v. 6, n. 4, 2016.

INÁCIO, R.F; BERNARDI, D; ROMANO, L.H. Análise comportamental do tecido adiposo frente ao tratamento de radiofrequência: revisão bibliográfica. Revista Saúde em Foco, n.9, 2017.

LOFEU, G.M. et al. Atuação da radiofrequência na gordura localizada no abdômen: revisão de literatura. Revista da Universidade Vale do Rio Verde, Três Corações, v.13, n.1, p.571-581, 2015.

MACHADO, A.T.O.M. et al. Benefícios da Massagem Modeladora na Lipodistrofia Localizada. Id on Line REVISTA DE PSICOLOGIA, v. 11, n. 35, p. 542-553, 2017.

MARTINS, C.R; PETROSKI, E.L. Insatisfação com a imagem corporal em adolescentes do sexo feminino de uma cidade de pequeno porte: prevalência e correlações. Motricidade, v. 11, n. 2, 2015. 
PUMPRLA, J. et al. Non-contact radiofrequency-induced reduction of subcutaneous abdominal fat correlates with initial cardiovascular autonomic balance and fat tissue hormones: safety analysis. F1000Research, v. 4, 2015.

SANTANA, M.LP et al. Factors associated with body image dissatisfaction among adolescents in public schools students in Salvador, Brazil. Nutrición hospitalaria, v. 28, n. 3, 2013.

SUH, D.H. et al. Safety and efficacy of a non-contact radiofrequency device for body contouring in Asians. Journal of Cosmetic and Laser Therapy, v. 19, n. 2, p. 89-92, 2017.

TAGLIOLATTO, S. Radiofrequência: método não invasivo para tratamento da flacidez cutânea e contorno corporal. Surgical \& Cosmetic Dermatology, v. 7, n. 4, 2015.

\section{Como citar este artigo (Formato ABNT):}

FERREIRA, Susan C.S.; MORAES, Karla C.S. de; BARROS, Jessica A.; SOUZA, Jussara S. de; SANTOS, Beatriz S. C.; FERREIRA, Juliana B. Ação e eficácia do tratamento com a Radiofrequência na adiposidade abdominal em mulheres. Id on Line Revista Multidisciplinar e de Psicologia, 2017, vol.11, n.38, p. 349-358. ISSN: 1981-1179.

Recebido: 30.10 .2017

Aceito: 31.10 .2017 